\title{
ON THE EMBEDDED PRIMARY COMPONENTS OF IDEALS (IV)
}

\author{
WILLIAM HEINZER, L. J. RATLIFF, JR., AND KISHOR SHAH
}

\begin{abstract}
The results in this paper expand the fundamental decomposition theory of ideals pioneered by Emmy Noether. Specifically, let $I$ be an ideal in a local ring $(R, M)$ that has $M$ as an embedded prime divisor, and for a prime divisor $P$ of $I$ let $I C_{P}(I)$ be the set of irreducible components $q$ of $I$ that are $P$-primary (so there exists a decomposition of $I$ as an irredundant finite intersection of irreducible ideals that has $q$ as a factor). Then the main results show: (a) $I C_{M}(I)=\bigcup\left\{I C_{M}(Q) ; Q\right.$ is a MEC of $\left.I\right\} \quad(Q$ is a MEC of $I$ in case $Q$ is maximal in the set of $M$-primary components of $I)$; (b) if $I=\bigcap\left\{q_{i} ; i=1, \ldots, n\right\}$ is an irredundant irreducible decomposition of $I$ such that $q_{i}$ is $M$-primary if and only if $i=1, \ldots, k<n$, then $\bigcap\left\{q_{i} ; i=1, \ldots, k\right\}$ is an irredundant irreducible decomposition of a MEC of $I$, and, conversely, if $Q$ is a MEC of $I$ and if $\bigcap\left\{Q_{j} ; j=1, \ldots, m\right\}$ (resp., $\bigcap\left\{q_{i} ; i=1, \ldots, n\right\}$ ) is an irredundant irreducible decomposition of $Q$ (resp., $I$ ) such that $q_{1}, \ldots, q_{k}$ are the $M$-primary ideals in $\left\{q_{1}, \ldots, q_{n}\right\}$, then $m=k$ and $\left(\bigcap\left\{q_{i} ; i=k+1, \ldots, n\right\}\right) \cap\left(\bigcap\left\{Q_{j} ; j=1, \ldots, m\right\}\right)$ is an irredundant irreducible decomposition of $I$; (c) $I C_{M}(I)=\{q ; q$ is maximal in the set of ideals that contain $I$ and do not contain $I: M\} ;$ (d) if $Q$ is a MEC of $I$, then $I C_{M}(Q)=\left\{q ; Q \subseteq q \in I C_{M}(I)\right\} ;(\mathrm{e})$ if $J$ is an ideal that lies between $I$ and an ideal $Q \in I C_{M}(I)$, then $J=\bigcap\left\{q ; J \subseteq q \in I C_{M}(I)\right\}$; and, (f) there are no containment relations among the ideals in $\bigcup\left\{I C_{P}(I) ; P\right.$ is a prime divisor of $I\}$.
\end{abstract}

\section{INTRODUCTION}

Our notation and terminology is mainly as in [Nag]. Background information may also be found in [Mat].

In the 1921 paper [N], Emmy Noether introduced irreducible decompositions of ideals (see [N, pp. 27-28]), and therein she proved the basic results concerning such decompositions of an ideal $I$ in a Noetherian ring. (Among other things, she showed that in any two such decompositions of $I$, the number of factors is the same [N, Satz IV] and the number of $P$-primary factors is the same for each prime divisor $P$ of $I$ [N, Satz VII].) On the other hand, in the three recent papers [HRS1, HRS2, HRS3] numerous results concerning the MECs of $I$ have been proved. The purpose of the present paper is to show the very close relationship (summarized in the abstract) between the irreducible components of $I$ that are $M$-primary and the irreducible components of the MECs of $I$.

Received by the editors September 3, 1993 and, in revised form, December 8, 1993; originally communicated to the Proceedings of the AMS by Wolmer V. Vasconcelos.

1991 Mathematics Subject Classification. Primary 13A17, 13C99; Secondary 13B99, 13 H99.

The first author's research on this paper was supported in part by the National Science Foundation, Grant DMS-9101176. 
In $\S 2$ we prove $(a)-(e)$ of the abstract, and (f) is proved in $\S 3$. The proofs of these results involve special cases of several of the results in [HRS1, HRS2, HRS3], so we specifically state these special cases in (2.2).

The authors have been fascinated by the historic and fundamental decomposition theorems of Emmy Noether, and this fascination gave rise to the theory of MECs as propounded in [HRS1, HRS2, HRS3] and the present paper. We are pursuing further topics in this new and fertile area of MECs, and we hope this theory turns out to be fascinating and useful to others.

\section{IRREDUCIBLE COMPONENTS AND MAXIMAL EMBEDDED COMPONENTS}

Throughout this section $(R, M)$ is a local ring and $I$ is an ideal that has $M$ as an embedded prime divisor. To show the very close connections between the irreducible components of $I$ and the irreducible components of the maximal embedded primary components of $I$ we need the following definitions and remark.

(2.1) Definition. Let $J$ be an ideal in a Noetherian ring $R$ and let $P$ be an embedded prime divisor of $J$. Then:

(2.1.1) $\mathbf{I C}_{\mathbf{P}}(\mathbf{J})$ denotes the set of irreducible $P$-primary ideals that appear in some decomposition of $J$ as an irredundant finite intersection of irreducible ideals. An ideal in $I C_{P}(J)$ is called an irreducible component of $J$.

(2.1.2) $\mathbf{D}_{\mathbf{P}}(\mathbf{J})$ denotes the number of $P$-primary ideals in a decomposition of $J$ as an irredundant finite intersection of irreducible ideals. (This number is well defined by [N, Satz VII].)

(2.1.3) If $R$ is local with maximal ideal $M$, and if $M$ is an embedded prime divisor of $J$, then a MEC (maximal embedded component) of $J$ is an $M$-primary ideal $Q$ that is maximal in the set of $M$-primary components of $J$.

(2.2) Remark. Let $I$ be an ideal in a local ring $(R, M)$ such that $M$ is an embedded prime divisor of $I$, let $V=I: M^{n}$ for large $n$, let $q$ be an $M$-primary component of $I$, and let $W$ be an $M$-primary ideal. Then the following hold:

(2.2.1) $I=V \cap W$ if and only if $W$ is an $M$-primary component of $I$.

(2.2.2) If $W \cap(I: M)=I$, then $W \cap V=I$.

(2.2.3) If $W$ is irreducible, $q \subseteq W$, and $q: W \nsubseteq M$, then

$$
l_{R}((q: M) /(W \cap(q: M)))=1
$$

(where $l_{R}(L)$ denotes the length of the $R$-module $L$ ).

(2.2.4) If $W_{1}, \ldots, W_{k}$ are ideals that contain $q$, then $W_{1} \cap \cdots \cap W_{k}=q$ if and only if $\left(W_{1} \cap \cdots \cap W_{k}\right) \cap(q: M)=q$.

(2.2.5) $q$ is a MEC of $I$ if and only if $(I: M) / I \cong(q: M) / q$.

(2.2.6) $D_{M}(q)=\operatorname{dim}_{R / M}((q: M) / q) \geq \operatorname{dim}_{R / M}((I: M) / I)$ and the strict inequality holds if and only if $q$ is not a MEC of $I$.

Proof. (2.2.1) follows immediately from the definitions, and (2.2.2) follows from the easily proved result: if $W \cap\left(I: M^{i}\right)=I$ for some $i \geq 1$, then $W \cap\left(I: M^{i+1}\right)=I$. Finally, (2.2.3)-(2.2.6) are proved in, respectively, [HRS3, (3.2)], [HRS3, (3.3)], [HRS2, (5.2)], and [HRS2, (5.2), (5.3), and (2.3.3)].

We can now state and prove the first of the main results in this section. 
(2.3) Theorem. Let $I$ be an ideal in a local ring $(R, M)$ that has $M$ as an embedded prime divisor. Then $I C_{M}(I)=\bigcup\left\{I C_{M}(Q) ; Q\right.$ is a MEC of $\left.I\right\}$. Moreover, if $I=\bigcap\left\{q_{i} ; i=1, \ldots, n\right\}$ is a decomposition of $I$ as an irredundant intersection of irreducible ideals with $q_{i} M$-primary if and only if $i=1, \ldots, k<n$, then $\bigcap\left\{q_{i} ; i=1, \ldots, k\right\}$ is an irredundant irreducible decomposition of a MEC $Q$ of $I$ and $D_{M}(Q)=D_{M}(I)=k$.

Proof. Let $q \in I C_{M}(I)$ and let $\bigcap\left\{q_{i} ; i=1, \ldots, n\right\}$ be a decomposition of $I$ as an irredundant intersection of irreducible ideals such that the $M$-primary ideals are the ideals $q_{1}, \ldots, q_{k}$ (for some $k<n$, so $D_{M}(I)=k$ ) and such that $q=q_{1}$. Also, let $Q=\bigcap\left\{q_{i} ; i=1, \ldots, k\right\}$ and let $V=I: M^{h}$ for large $h$, so $V=\left(\bigcap\left\{q_{i} ; i=1, \ldots, n\right\}\right): M^{h}=\bigcap\left\{q_{i} ; i=k+1, \ldots, n\right\}$ is the isolated component of $I$ determined by $R-\bigcup\{P ; P \in \operatorname{Ass}(R / I)$ and $P \neq M\}$. Then $Q \cap V=I$, so it follows from (2.2.1) that $Q$ is an $M$-primary component of $I$, and it will now be shown that $Q$ is, in fact, a MEC of $I$ and that $\bigcap\left\{q_{i} ; i=1, \ldots, k\right\}$ is a decomposition of $Q$ as an irredundant intersection of irreducible ideals (so $q=q_{1} \in I C_{M}(Q)$ and $D_{M}(Q)=k=D_{M}(I)$ ), as desired).

For this, let $Q^{*}$ be a MEC of $I$ such that $Q \subseteq Q^{*}$ and let $\bigcap\left\{Q_{j} ; j=\right.$ $1, \ldots, p\}$ be a decomposition of $Q^{*}$ as an irredundant intersection of irreducible ideals. Then (2.2.6) shows that $p \leq k$ and that the equality holds if and only if $Q$ is a MEC of $I$ (and then $Q=Q^{*}$ ). Therefore, since $Q=\bigcap\left\{q_{i} ; i=1, \ldots, k\right\}$ is an intersection of irreducible ideals, if $p=k$, then it follows that $\bigcap\left\{q_{i} ; i=1, \ldots, k\right\}$ is, in fact, a decomposition of $Q$ as an irredundant intersection of irreducible ideals. Therefore it suffices to show that $p=k$.

For this, $I=\left(\cap\left\{q_{i} ; i=1, \ldots, n\right\}\right)=Q \cap V=Q^{*} \cap V$ (by (2.2.1)) $=\left(\bigcap\left\{Q_{j} ; j=1, \ldots, p\right\}\right) \cap\left(\bigcap\left\{q_{i} ; i=k+1, \ldots, n\right\}\right)$. But $\left(\bigcap\left\{Q_{j} ; j=\right.\right.$ $1, \ldots, p\}) \cap\left(\bigcap\left\{q_{i} ; i=k+1, \ldots, n\right\}\right)$ is an intersection of irreducible ideals (that equals $I$ ), so $p+(n-k) \geq n$ (since each decomposition of $I$ as an intersection of irreducible ideals must have at least $n$ factors). Therefore $p \geq k$, and it has already been noted that $p \leq k$, hence $p=k$, so $I C_{M}(I) \subseteq \bigcup\left\{I C_{M}(Q) ; Q\right.$ is a MEC of $I\}$.

For the opposite inclusion let $Q^{*}$ be a MEC of $I$ and let $q \in I C_{M}\left(Q^{*}\right)$, so there exists a decomposition $\bigcap\left\{Q_{j} ; j=1, \ldots, t\right\}$ of $Q^{*}$ as an irredundant intersection of irreducible ideals such that $q=q_{1}$. Let $\bigcap\left\{q_{i} ; i=1, \ldots, n\right\}$ be a decomposition of $I$ as an irredundant intersection of irreducible ideals such that the $M$-primary ideals are the ideals $Q_{1}, \ldots, q_{k}$ (for some $k<n$ ) and let $Q=\bigcap\left\{q_{i} ; i=1, \ldots, k\right\}$, so $Q$ is also a MEC of $I$ (as already shown). Therefore $k=t$, since $D_{M}\left(Q^{\prime}\right)$ is the same for all MECs $Q^{\prime}$ of $I$ by (2.2.6). Let $V$ be as in the first paragraph of this proof. Then $I=V \cap Q^{*}$ (by $(2.2 .1))=\left(\bigcap\left\{q_{i} ; i=k+1, \ldots, n\right\}\right) \cap\left(\bigcap\left\{Q_{j} ; j=1, \ldots, t\right\}\right)$ is an intersection of irreducible ideals (that equals $I)$ that has $t+(n-k)=n$ factors, so it is an irredundant intersection (since each decomposition of $I$ as an intersection of irreducible ideals must have at least $n$ factors). Therefore, since $q=Q_{1}$, it follows that $I C_{M}\left(Q^{*}\right) \subseteq I C_{M}(I)$. It then follows that $\bigcup\left\{I C_{M}(Q) ; Q\right.$ is a MEC of $I\} \subseteq I C_{M}(I)$, so it follows from the first part of this proof that $\bigcup\left\{I C_{M}(Q) ; Q\right.$ is a MEC of $\left.I\right\}=I C_{M}(I)$.

(2.4) Corollary. Let $Q^{*}$ be a MEC of $I$, let $\bigcap\left\{Q_{j} ; j=1, \ldots, m\right\}$ (resp., $\bigcap\left\{q_{i} ; i=1, \ldots, n\right\}$ ) be an irredundant irreducible decomposition of $Q^{*}$ (resp., 
$I)$, and assume that $q_{1}, \ldots, q_{k}$ are the $M$-primary ideals in $\left\{q_{1}, \ldots, q_{n}\right\}$. Then $m=k$ and $\left(\bigcap\left\{q_{i} ; i=k+1, \ldots, n\right\}\right) \cap\left(\bigcap\left\{Q_{j} ; j=1, \ldots, m\right\}\right)$ is an irredundant irreducible decomposition of $I$.

Proof. This was proved in the last paragraph of the proof of (2.3).

To extend (2.3) to an ideal $I$ in a Noetherian (not necessarily local) ring $R$ we need the following definition.

(2.5) Definition. Let $P$ be an embedded prime divisor of an idea $J$ in a Noetherian ring $R$. Then a P-MEC of $J$ is a $P$-primary component of $J$ that is not properly contained in any other $P$-primary component of $J$.

(2.6) Remark. In [HRS3, (6.2)] it is shown that there exists a one-to-one correspondence between the $P$-MECs $q$ of $J$ and the MECs $Q$ of $J R_{P}$ given by $Q=q R_{P}$ and $q=Q \cap R$.

(2.7) Theorem. Let $J=\bigcap\left\{q_{i} ; i=1, \ldots, n\right\}$ be an irredundant irreducible decomposition of an ideal $J$ in a Noetherian ring $R$, let $P$ be an embedded prime divisor of $J$, and assume that $q_{i}$ is $P$-primary if and only if $i=1, \ldots, h<n$. Then $\bigcap\left\{q_{i} ; i=1, \ldots, h\right\}$ is an irredundant irreducible decomposition of a $P$-MEC $Q$ of $J, D_{P}(Q)=D_{P}(J)=h$, and $\bigcup\left\{I C_{P}(q) ; q\right.$ is a P-MEC of $J\}=I C_{P}(J)$.

Proof. This follows immediately from (2.3) and (2.6).

A special case of the next corollary of (2.3) and (2.7) characterizes when an $M$-primary component of $J$ is a MEC of $J$.

(2.8) Corollary. Let $J, R$, and $P$ be as in (2.7) and let $Q$ be a P-primary component of $J$. Then $Q$ is a P-MEC of $J$ if and only if $Q$ is the intersection of $D_{P}(J)$ ideals in $I C_{P}(J)$. If $Q$ is a $P$-MEC of $J$, if $\bigcap\left\{Q_{j} ; j=1, \ldots, m\right\}$ (resp., $\bigcap\left\{q_{i} ; i=1, \ldots, n\right\}$ ) is an irredundant irreducible decomposition of $Q$ (resp., $J R_{P} \cap R$ ), and if $q_{1}, \ldots, q_{h}$ are the $P$-primary ideals in $\left\{q_{1}, \ldots, q_{n}\right\}$, then $m=h$ and $\left(\bigcap\left\{q_{i} ; i=h+1, \ldots, n\right\}\right) \cap\left(\bigcap\left\{Q_{j} ; j=1, \ldots, m\right\}\right)$ is an irredundant irreducible decomposition of $J R_{P} \cap R$.

Proof. By (2.6) it may be assumed that $R$ is local with maximal ideal $M=P$. Then $Q$ is the intersection of $D_{M}(Q)$ ideals in $I C_{M}(Q)$, so if $Q$ is a MEC of $J$, then (2.3) shows that $Q$ is the intersection of $D_{M}(J)=D_{M}(Q)$ ideals in $I C_{M}(J)$.

For the converse, if $Q$ is the intersection of $D_{M}(J)$ ideals in $I C_{M}(J)$, then $D_{M}(Q) \leq D_{M}(J)$. However, $D_{M}(J)=D_{M}\left(Q^{*}\right)$ for some MEC $Q^{*}$ of $J$, by (2.3), and $D_{M}\left(Q^{*}\right) \leq D_{M}\left(Q^{\prime}\right)$ for each $M$-primary component $Q^{\prime}$ of $J$ and the equality holds if and only if $Q^{\prime}$ is a MEC of $J$ by (2.2.6). Therefore, since $Q$ is an $M$-primary component of $J$, it follows that $D_{M}(J) \leq D_{M}(Q)$, so $D_{M}(Q)=D_{M}(J)$, hence $Q$ is a MEC of $J$.

The final statement follows from (2.4).

In (2.9) we characterize the ideals in $I C_{M}(I)$. This result (and its related (2.10.1)) is then used in (2.12) to show that each ideal between $I$ and an ideal in $I C_{M}(I)$ is the intersection of the ideals in $I C_{M}(I)$ that contain it.

(2.9) Proposition. Let $(R, M)$ and $I$ be as in (2.3) and let $S=\{q ; q$ is an ideal in $R, I \subseteq q$, and $I: M \nsubseteq q\}$. Then $I C_{M}(I)=\{q ; q$ is maximal in $S\}$. 
Proof. Let $q$ be maximal in $S$ and let $K=(q / I) \cap((I: M) / I)$. Then $K$ is a proper subspace of the $R / M$-vector space $(I: M) / I$, so let $e_{1}, \ldots, e_{d}$ be a basis of $K$ and extend it to a basis $e_{1}, \ldots, e_{d}, e_{d+1}, \ldots, e_{k}$ of $(I: M) / I$. For $i=1, \ldots, k$ let $x_{i}$ be a pre-image in $R$ of $e_{i}$. Then since $q$ is maximal in $S$ it follows that $k=d+1$ (hence $\left.\operatorname{dim}_{R / M}((I: M) / I)=d+1\right)$. Also, if $q_{i}$ is maximal in $S_{i}=\left\{H ; H\right.$ is an ideal in $R,\left(I, x_{1}, \ldots, x_{i-1}, x_{i+1}, \ldots, x_{d+1}\right) R \subseteq H$, and $\left.x_{i} \notin H\right\}$, then: $q_{i}$ is irreducible (since every ideal that properly contains $q_{i}$ must contain $\left.x_{i}\right) ; q_{i}$ is $M$-primary (since $q_{i}+M^{n} \in S_{i}$ for all large $n$ ); and, $\left(q_{1} \cap \cdots \cap q_{d+1}\right) \cap(I: M)=I$. Further, $q \in S_{d+1}$ and $q$ is maximal in $S$, so every ideal $J$ that properly contains $q$ must also contain $I: M$, so $x_{d+1} \in J$, so we may choose $q_{d+1}$ to be $q$. Finally, let $Q=\bigcap_{j=1}^{d+1} q_{j}$ (so $Q$ is $M$-primary and $D_{M}(Q) \leq d+1$ ) and let $V=I: M^{n}$ for large $n$. Then $Q \cap(I: M)=I$, as just noted, so $Q \cap V=I$, by (2.2.2), so $Q$ is an $M$-primary component of $I$ (by (2.2.1)). Therefore, (2.2.6) shows that $D_{M}(Q) \geq \operatorname{dim}_{R / M}((I: M) / I)=d+1$, so $D_{M}(Q)=d+1$. Therefore $Q$ is a MEC of $I$ (by (2.2.6)) and $q=q_{d+1} \in I C_{M}(Q)$, so $q \in I C_{M}(I)$, by (2.3), hence $\{q ; q$ is maximal in $S\} \subseteq I C_{M}(I)$.

For the opposite inclusion let $q \in I C_{M}(I)$. Then (2.3) shows that $q \in$ $I C_{M}(Q)$ for some MEC $Q$ of $I$. Let $Q=q_{1} \cap \cdots \cap q_{n}$ be an irredundant irreducible decomposition of $Q$ such that $q=q_{1}$. For $i=1, \ldots, n$ let $H_{i}=q_{i} \cap(Q: M)$, so $H_{1} \cap \cdots \cap H_{n}$ is an irredundant decomposition of $Q$, by (2.2.4), so $q_{i} \cap(Q: M)$ is properly contained in $Q: M$ for $i=1, \ldots, n$. Also, $(I: M) / I \cong(Q: M) / Q$, by (2.2.5), and the natural isomorphism $f$ is given by $f(x+I)=x+Q$, where $x \in I: M$, so it follows that $q_{i} \cap(I: M)$ is properly contained in $I: M$ for $i=1, \ldots, n$. In particular, since $q=q_{1}$ it follows that $I: M \nsubseteq q$. Therefore $q \in S$, and if $q^{\prime}$ is an ideal that properly contains $q$, then $q: M \subseteq q^{\prime}$ (since $q: M$ is the unique ideal that is minimal with respect to properly containing $q$ ). Therefore $I: M \subseteq Q: M \subseteq q: M \subseteq q^{\prime}$, so $q$ is maximal in $S$, hence $I C_{M}(I) \subseteq\{q ; q$ is maximal in $S\}$.

(2.10) Remark.

(2.10.1) If $J$ is an ideal containing $I$, if $x \in(I: M)-J$, and if $q$ is maximal in $T=\{H ; H$ is an ideal in $R, J \subseteq H$, and $x \notin H\}$, then $q \in I C_{M}(I)$.

(2.10.2) If $P$ is an embedded prime divisor of $I$, then $I C_{P}(I)=\{q ; q$ is maximal in $U\}$, where $U=\{H ; H$ is an ideal in $R, I \subseteq H \subseteq P$, and $I: P \nsubseteq H\}$.

(2.10.3) If $J$ is an ideal such that $I \subseteq J \subseteq P$, if $x \in(I: P)-J$, and if $q$ is maximal in $V=\{H ; H$ is an ideal in $R, J \subseteq H \subseteq P$, and $x \notin H\}$, then $q \in I C_{P}(I)$.

Proof. For (2.10.1), $I \subseteq q$ and $I: M \nsubseteq q$, so $q$ is in the set $S$ of (2.9). Also, $q$ is irreducible (since every ideal that properly contains $q$ must contain $x$ ) and $M$-primary (since $q+M^{n} \in T$ for all large $n$ ), so $q: M$ is the unique ideal that is minimal with respect to properly containing $q$. Therefore $I: M$ is contained in every ideal that properly contains $q$ (since $I: M \subseteq q: M)$, so $q$ is maximal in the set $S$ of (2.9), hence $q \in I C_{M}(I)$ by (2.9). $P$.

(2.10.2) (resp., (2.10.3)) follows from (2.9) (resp., (2.10.1)) by localizing at

In (2.11) we characterize when an ideal in $I C_{M}(I)$ is in $I C_{M}(Q)$ for some $\operatorname{MEC} Q$ of $I$. 
(2.11) Proposition. Let $R, J$, and $P$ be as in (2.7), let $Q$ be a P-MEC of $J$, and let $q \in I C_{P}(J)$. Then $q \in I C_{P}(Q)$ if and only if $Q \subseteq q$.

Proof. It is clear that if $q \in I C_{P}(Q)$, then $Q \subseteq q$.

For the converse, by (2.6) it may be assumed that $R$ is local with maximal ideal $M=P$. Therefore assume that $Q \subseteq q$. Then $J: M \nsubseteq q$, by (2.9) (since $\left.q \in I C_{M}(J)\right)$, so $Q: M \nsubseteq q$. Therefore $K=(q / Q) \cap((Q: M) / Q)$ is a proper subspace of the $R / M$-vector space $(Q: M) / Q$, so the proof that $q \in I C_{M}(Q)$ is similar to the first paragraph of the proof of (2.9) (using the fact that, since $q$ is irreducible, $k=d+1$ (by (2.2.3))).

In [HRS1, (2.15)] it is shown that if $Q$ is a MEC of $I$ and $J$ is an ideal between $I$ and $Q$, then $J$ is the intersection of the MECs of $I$ that contain $J$. (2.12) shows that the analogous result holds when "MEC" is replaced with "irreducible component".

(2.12) Theorem. Let $I$ and $(R, M)$ be as in (2.3) and let $J$ be an ideal between $I$ and an ideal $Q \in I C_{M}(I)$. Then $J=\bigcap\left\{q ; J \subseteq q \in I C_{M}(I)\right\}$.

Proof. Assume that the conclusion holds when $J$ is $M$-primary. Then $J=$ $\bigcap\left\{J+M^{n} ; n \geq 1\right\}$ and $J+M^{n}=\bigcap\left\{q ; J+M^{n} \subseteq q \in I C_{M}(I)\right\}$ (by the assumption), so $J=\bigcap\left\{q ; J \subseteq q \in I C_{M}(I)\right\}$. Therefore it may be assumed that $J$ is $M$-primary.

Let $K=\bigcap\left\{q ; J \subseteq q \in I C_{M}(I)\right\}$, so $J \subseteq K$ and it remains to show that $J=$ $K$. For this, it will first be shown that:

(2.12.1) $J \cap(I: M)=K \cap(I: M) \subset I: M$.

For this, $I: M \nsubseteq q$ for each $q \in I C_{M}(I)$, by (2.9), so $I: M \nsubseteq \bigcap\{q ; J \subseteq$ $\left.q \in I C_{M}(I)\right\}=K$, so $K \cap(I: M) \subset I: M$. Now suppose there exists $x \in$ $K \cap(I: M), \notin J \cap(I: M)$, and let $q^{*}$ be maximal in $\{H ; H$ is an ideal in $R, J \subseteq H$, and $x \notin H\}$. Then $q^{*} \in I C_{M}(I)$, by (2.10.1), and $J \subseteq q^{*}$. Therefore $x \in K=\bigcap\left\{q ; J \subseteq q \in I C_{M}(I)\right\} \subseteq q^{*}$, and this is a contradiction, so $J \cap(I: M)=K \cap(I: M)$, hence $(2.12 .1)$ holds.

Now suppose that $J$ is a proper subset of $K$. Then since $J$ is $M$-primary, there exists $x \in K-J$ such that $x M \subseteq J$. Also, by (2.12.1) there exists $y \in(I: M)-K$, so let $K^{\prime}=(J, x+y) R$. Then it will next be shown that:

(2.12.2) $J \cap(I: M)=K^{\prime} \cap(I: M)$.

For this, suppose there exists $z \in\left(K^{\prime} \cap(I: M)\right)-(J \cap(I: M))$, so $z=$ $j+r(x+y)$ for some $j \in J$ and for some $r \in R$. Then $r$ is a unit, since $(x+y) M \subseteq J$ and $z \notin J$. Also, $z-r y=j+r x \in K \cap(I: M)=J \cap(I: M)$ (by (2.12.1)). Therefore $j+r x \in J \cap(I: M) \subseteq J, j \in J$, and $r$ is a unit, hence $x \in J$, and this contradicts the choice of $x$. Therefore (2.12.1) holds.

Now, since $K \cap(I: M)=J \cap(I: M)=K^{\prime} \cap(I: M)$, by (2.12.1) and (2.12.2), and since $y \in(I: M)-K$, there exists an ideal $q^{\prime}$ that contains $K^{\prime}$ and is maximal with respect to not containing $y$, and then $q^{\prime} \in I C_{M}(I)$ by $(2.10 .1)$. Also, $J \subseteq K^{\prime} \subseteq q^{\prime}$, so $K \subseteq q^{\prime}$ by the definition of $K$. Therefore $x+y \in K^{\prime} \subseteq q^{\prime}$ and $x \in K \subseteq q^{\prime}$, so $y \in q^{\prime}$, and this contradicts the definition of $q^{\prime}$, hence $J=K$.

(2.13) sharpens the conclusion of (2.12) when $J$ is an ideal between a MEC $Q^{*}$ of $I$ and an ideal $q \in I C_{M}(I)$. (Concerning the existence of $Q^{*}$ in (2.13), 
since $Q \in I C_{M}(I)$, there exists a MEC $Q^{*}$ of $I$ such that $Q \in I C_{M}\left(Q^{*}\right)$ by (2.3).)

(2.13) Corollary. Let $(R, M)$ and $I \subseteq J \subseteq Q \in I C_{M}(I)$ be as in (2.12). Let $Q^{*}$ be a MEC of $I$ such that $Q \in I C_{M}\left(Q^{*}\right)$ and assume that $Q^{*} \subseteq J$. Then $J=\bigcap\left\{q ; J \subseteq q \in I C_{M}\left(Q^{*}\right)\right\}$.

Proof. $J=\bigcap\left\{q ; J \subseteq q \in I C_{M}(I)\right\}$ by (2.12). Also, since $Q^{*} \subseteq J$, each ideal $q \in I C_{M}(I)$ that contains $J$ is in $I C_{M}\left(Q^{*}\right)$, by (2.11), so $J=\bigcap\{q ; J \subseteq q \in$ $\left.I C_{M}\left(Q^{*}\right)\right\}$.

\section{THERE ARE NO CONTAINMENT RELATIONS AMONG THE IRREDUCIBLE COMPONENTS OF AN IDEAL}

The title of this section is its main result. That is, in (3.2) we show that there are no containment relations among the ideals in the various finite irredundant irreducible decompositions of an ideal in a Noetherian ring. To prove this we need the following Irreducibility Lemma of Emmy Noether. (The "only if" part is stated and proved in [N, Hilfsatz II]; reading [N], one gets the feeling that she knew the converse is also true.)

(3.1) Lemma. Let $I$ be an ideal in a ring $R$. Then $I$ is irreducible if and only if $I \cap K \subset I^{\prime} \cap K$ for all ideals $K$ not contained in $I$ and for all ideals $I^{\prime}$ that properly contains $I$.

Proof. Assume first that $I$ is irreducible, let $I^{\prime}$ be an ideal that properly contains $I$, let $K$ be an ideal that is not contained in $I$, let $a \in I^{\prime}-I$, and let $b \in K-I$. Then since $I$ is irreducible, there exists an element $c \in((I, a) R \cap(I, b) R)-I$, so $c=j-r b$ for some $j \in I$ and for some $r \in R$. Then $c-j=r b \in\left(I^{\prime} \cap K\right)-(I \cap K)$, so $I \cap K \subset I^{\prime} \cap K$.

For the converse, assume that $I \cap K \subset I^{\prime} \cap K$ for each ideal $I^{\prime}$ that properly contains $I$ and for each ideal $K$ that is not contained in $I$. Suppose that $I$ is not irreducible, so there exist ideals $I^{\prime}$ and $K$ such that $I \subset I^{\prime}, I \subset K$, and $I=I^{\prime} \cap K$. But by hypothesis we have $I \cap K \subset I^{\prime} \cap K$, so $I=I \cap K \subset I^{\prime} \cap K=I$, and this is a contradiction. Therefore $I$ is irreducible.

(3.2) Theorem. Let $J$ be an ideal in a Noetherian ring $R$, let $P_{1}, \ldots, P_{m}$ be the prime divisors of $J$, and let $I C(J)=I C_{P_{1}}(J) \cup \cdots \cup I C_{P_{m}}(J)$. Then there are no containment relations among the ideals in $I C(J)$.

Proof. Suppose there exist ideals $q$ and $Q$ in $I C(J)$ such that $q \subset Q$. Then there exist finite irredundant irreducible decompositions: $\left(^{*}\right) J=\bigcap_{i=1}^{n} q_{i}$ (with $q=q_{1}$ ); and ( $\left.{ }^{* *}\right) J=\bigcap_{i=1}^{n} Q_{i}$ (with $Q=Q_{1}$ ). Then $J=\bigcap_{i=1}^{n} Q_{i} \supseteq q_{1} \cap$ $\left(\bigcap_{i=2}^{n} Q_{i}\right) \supseteq J$, so: $\left({ }^{* * *}\right) J=q_{1} \cap\left(\bigcap_{i=2}^{n} Q_{i}\right)$ is an irredundant irreducible decomposition of $J$ (since there are $n$ factors). Therefore it follows from $\left({ }^{* *}\right)$ and (***) that: (\#) $Q_{1} \cap\left(\bigcap_{i=2}^{n} Q_{i}\right)=J=q_{1} \cap\left(\bigcap_{i=2}^{n} Q_{i}\right)$. However, (\#) contradicts Noether's Irreducibility Lemma (3.1) (with $I=q_{1}, I^{\prime}=Q_{1}$, and $\left.K=\bigcap_{i=2}^{n} Q_{i}\right)$. Therefore there are no containment relations among the ideals in $I C(J)$. 


\section{REFERENCES}

[HRS1] W. Heinzer, L. J. Ratliff, Jr., and K. Shah, On the embedded primary components of ideals (I), J. Algebra 167 (1994), 724-744.

[HRS2] _ On the embedded primary components of ideals (II), J. Pure Appl. Algebra (to appear).

[HRS3] _ On the embedded primary components of ideals (III), J. Algebra (to appear).

[Mat] H. Matsumura, Commutative ring theory, Cambridge Studies in Advanced Mathematics, No. 8, Cambridge Univ. Press, Cambridge, 1986.

[N] E. Noether, Idealtheorie in Ringbereichen, Math. Ann. 83 (1921), 24-66.

[Nag] M. Nagata, Local rings, Interscience Tracts No. 13, Interscience, New York, 1961.

Department of Mathematics, Purdue University, West Lafayette, Indiana 47907

E-mail address: heinzer@math.purdue.edu

Department of Mathematics, University of California, Riverside, California 92521

E-mail address: ratliff@ucrmath.ucr.edu

Department of Mathematics, Southwest Missouri State University, Springfield, MisSOURI 65804

E-mail address: kis100f Qvma.smsu.edu 